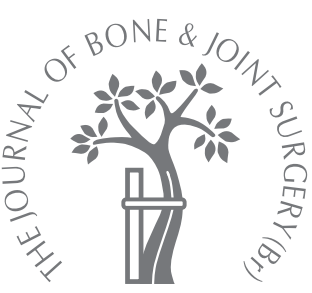

N. Lubega, N. C. Mkandawire, G. C. Sibande, A. R. Norrish, W. J. Harrison

From Beit Cure Hospital, Blantyre, Malawi

\title{
Joint replacement in Malawi
}

\author{
ESTABLISHMENT OF A NATIONAL JOINT REGISTRY
}

In Africa the amount of joint replacement surgery is increasing, but the indications for operation and the age of the patients are considerably different from those in the developed world. New centres with variable standards of care and training of the surgeons are performing these procedures and it is important that a proper audit of this work is undertaken.

In Malawi, we have pioneered a Registry which includes all joint replacements that have been carried out in the country. The data gathered include the age, gender, indication for operation, the prosthesis used, the surgical approach, the use of bone graft, the type of cement, pressurising systems and the thromboprophylaxis used. All patients have their clinical scores recorded pre-operatively and then after three and six months and at one year. Before operation all patients are counselled and on consent their HIV status is established allowing analysis of the effect of HIV on successful joint replacement.

To date, 73 total hip replacements (THRs) have been carried out in 58 patients by four surgeons in four different hospitals. The most common indications for THR were avascular necrosis (35 hips) and osteoarthritis (22 hips). The information concerning 20 total knee replacements has also been added to the Registry.

Africa is unique in many aspects of joint replacement. There are different indications for the procedure, the patients are younger and there are different factors in life style which may affect the longevity of the prosthesis. The long-term effect of the presence of HIV infection requires further investigation.

Patients infected with HIV may be at risk of late deep infection of the implant as their immune status deteriorates. ${ }^{1}$ Harrison, Lewis and Lavy ${ }^{2}$ have suggested removal of trauma implants soon after union of the fracture if infection occurs. However, these studies were carried out before the widespread use of highly active antiretroviral treatment (HAART) in sub-Saharan Africa. Implants cannot be removed without affecting function. A large retrospective multicentre study by Hicks et $\mathrm{al}^{3}$ showed that there was an increased rate of sepsis after joint replacement in HIV-positive haemophiliacs. We are uncertain about late sepsis in HIV-positive patients especially those given HAART and prophylactic cotrimoxazole. In Malawi the present criterion for starting patients on HAART is a CD4 count which is lower than $250 \times 10^{6} / \mathrm{l}$, but the question remains as to whether HAART reduces the risk of late sepsis, and, if so, whether this is simply by elevating the CD4 count. Long-term followup of all HIV-positive patients who have undergone joint replacement should ascertain whether deep infection occurs as immunity deteriorates.

\section{Patients and Methods}

The National Joint Registry was established in 2005 with ethical approval from the Research and Ethics Committee of the College of Medicine of Malawi (Table I). All patients who have had or are about to undergo total hip replacement (THR) in Malawi are approached to give consent to be included in the Registry. To date 73 THRs have been entered, together with 20 total knee replacements.

An assessment is carried out one week before the operation by a senior nurse (GCS). This includes calculating the Harris hip score ${ }^{4}$ (HHS) and obtaining informed consent from the patient for both the procedure and inclusion in the Registry. After counselling and consent, HIV testing is carried out, with determination of the CD4 level in those who test positive. Routine pre-operative tests include a full blood count and a blood slide for malaria parasites. All questions pertaining to the procedure are answered and general counselling is given so that the 
Table I. Data collected for the Malawi National Joint Registry

Age

Gender

Surgical indication (primary osteoarthritis, $A V N^{*}$ )

Surgeon's grade (training or consultant surgeon)

Hospital where surgery performed

Type of anaesthesia used (regional or general)

ASA grade (American Society of Anesthesiologists, grades 1 to 5)

Body mass index

Surgical approach

Type of implant

Cementing technique

HIV serostatus of the patient (with CD4 count for seropositive patients) Harris hip score (pre- and post-operative)

* AVN, avascular necrosis

Table II. Indications for total hip replacement (THR)

\begin{tabular}{ll}
\hline & Number of hips \\
\hline Primary THR & 35 \\
AVN & 22 \\
Primary osteoarthritis & 7 \\
Intracapsular fracture of the neck of the femur & 3 \\
Previous fracture/dislocation of the hip & 2 \\
Perthes' disease, secondary osteoarthritis & 1 \\
AVN due to sickle-cell disease & 1 \\
Giant-cell tumour & 1 \\
Previous tuberculosis of the hip & \\
& \\
Revision THR & 1 \\
Aseptic loosening (at six years)
\end{tabular}

* AVN, avascular necrosis

patient is primed on what to expect after the operation. The patients are then admitted on the day before surgery.

After operation, all patients are reviewed at six weeks, three and six months and then annually. They are assessed for pain and function using the HHS. Radiographs are evaluated for radiolucencies or fractures around the implants, a change in their position and heterotopic ossification. HIVpositive patients have their CD4 count rechecked annually and a record made as to the date on which they started HAART, if this is relevant. Those with a CD4 count of less than $250 \times 10^{6}$ cells/l are given HAART. The patients who decline to have HIV tests at the time of surgery are offered voluntary counselling and testing at each review.

Of the 73 THRs in 58 patients 15 were staged bilateral procedures. These patients were operated on by four different surgeons in four different hospitals. No hospital had a laminar-flow theatre. There were 40 operations in 33 men (seven bilateral) and 33 in 25 women (eight bilateral). The mean age of the patients at the time of surgery was 52 years (18 to 73 ) and the mean weight $78 \mathrm{~kg}$ (48 to 111$)$.

The indications for surgery for the patients are shown in Table II. One had THR secondary to sickle-cell disease associated with avascular necrosis (AVN) of the head of the femur. He was the youngest in our series, being aged 18 years at the time of operation. Another aged 22 years had been treated for tuberculosis of the hip two years before THR. He was given prophylactic treatment for tuberculosis after THR to prevent possible reactivation of the infection.

Statistical analysis. The parametric data was analysed using the Student's $t$-test.

\section{Results}

The HIV status of each of the patients receiving a THR is shown in Table III. Of the 18 patients who were HIVpositive, $14(77.8 \%)$ had $\mathrm{AVN}$ as the indication for operation compared with $14(36.8 \%)$ of the 38 patients in the HIV-seronegative group. The serostatus of 17 patients was unknown and AVN was the indication for THR in seven $(41.2 \%)$ of these patients.

The mean pre-operation CD4 count in the 18 HIVpositive patients was $543 \times 10^{6}$ cells/l (183 to 1410$)$. One had a CD4 of $86 \times 10^{6}$ cells/l when seen after two years which six months later had decreased to $30 \times 10^{6}$ cells/l. He had been on first-line HAART one year before THR. He has been referred to the HIV specialist for assessment with a view to changing to second-line HAART. One patient was placed on second-line HAART in May 2007 after her CD4 count was falling despite being on first-line HAART from 2004. She had a THR in July 2006 and her CD4 count at the last follow-up in April 2008 was $507 \times 10^{6}$ cells $/$.

Charnley prostheses with either a flanged or roundback femoral stem and a standard long posterior wall or Ogee acetabular component (Depuy) were used in 43 patients and Stanmore implants with modular stems and $28 \mathrm{~mm}$ cobaltchrome heads (Biomet) were used in 29. One patient had a metal-on-ceramic implant. All the implants were cemented mostly using Smartset GHV with gentamicin (Depuy). All patients received cefuroxime $(1500 \mathrm{mg})$ intravenously at the time of induction and three doses of $750 \mathrm{mg}$ of cefuroxime post-operatively.

For thromboprophylaxis all patients had thromboembolus detergent (TED) graduated compression stockings and 49 received low-dose aspirin for a period of six weeks after operation. A further 22 received low-molecular-weight heparin for a mean of five days after surgery and two had no chemical treatment. All patients were mobilised on the first day after operation.

The mean pre-operative HHS was 29 (9 to 59) and the mean post-operative score 85 (79 to 91 ), giving a mean improvement of 56 points ( $\mathrm{p}<0.05$, Student's $t$-test). The mean number of days spent in hospital was ten ( 7 to 19). The patient who spent 19 days in hospital had a superficial wound infection which cleared with antibiotic treatment.

The patient with tuberculosis was free from infection at follow-up at two years.

Complications. There were no cases of deep sepsis. About six weeks after operation two patients dislocated the hip which was successfully reduced without further recurrence. One patient had a deep-vein thrombosis and subsequent pulmonary embolism and was given a prolonged course of anticoagulants. Another patient had aseptic loosening after six years and required revision. He was 54 years old at the initial 
Table III. HIV status of the patients undergoing total hip replacement (THR)

\begin{tabular}{|c|c|c|c|c|}
\hline & \multicolumn{3}{|c|}{ HIV $^{*}$ serostatus } & \multirow[b]{2}{*}{ Number of patients } \\
\hline & Negative & Positive & Unknown & \\
\hline \multicolumn{5}{|l|}{ Primary THR } \\
\hline $\mathrm{AVN}^{\dagger}$ & 11 & 10 & 4 & 25 \\
\hline Primary osteoarthritis & 7 & & 11 & 18 \\
\hline Intracapsular fracture of the neck of the femur & 4 & 2 & 1 & 7 \\
\hline Previous fracture/dislocation of the hip & 1 & 1 & & 2 \\
\hline Perthes' disease - secondary osteoarthritis & 1 & 1 & & 2 \\
\hline Sickle-cell AVN & 1 & & & 1 \\
\hline Giant-cell tumour & 1 & & & 1 \\
\hline Previous tuberculosis of the hip & 1 & & & 1 \\
\hline \multicolumn{5}{|l|}{ Revision THR } \\
\hline Aseptic loosening (at six years) & 1 & & & 1 \\
\hline Total & 28 & 14 & 16 & 58 \\
\hline
\end{tabular}

operation for AVN subsequent to a fracture of the neck of the femur. There have been no other cases of clinical or radiological loosening. There was early setting of the cement in one patient before the femoral stem had been fully inserted requiring revision of the component.

\section{Discussion}

The early results of THR in Malawi are encouraging and are comparable with those in other series in regard to complications and improvement in pain and function.

The Swedish Registry reported that between 1992 and 1994 the mean age of patients undergoing primary THR was 70 years. ${ }^{5}$ In Malawi the mean age of the patients was 52 years. We need to know the survival of these hips in relatively young patients and whether we should begin to use cementless arthroplasty or even resurfacing in them.

The indications for the procedure in our patients were different from those in Sweden where primary osteoarthritis $(78 \%)$ and osteoarthritis secondary to trauma $(11 \%)$ are the main reasons for operation. ${ }^{5}$ However, in Malawi 35 of the 73 hips $(47.9 \%)$ had THR for AVN. It is not clear whether there is an association between AVN of the femoral head as a result of HIV disease or HAART or both.

Our early results showed that it was safe to carry out joint replacement in HIV patients as long as the skin was intact and the conditions ideal. ${ }^{2}$ There was no difference in early infection between patients who were HIV-positive and those who were HIV-negative. We do not yet know whether late sepsis may occur around joint replacements in HIV-positive patients, and the role of HAART is also uncertain. Hicks et $\mathrm{al}^{3}$ found a high incidence of late sepsis around joint replacements in haemophiliacs, but Harrison ${ }^{6}$ noted that these results might not be representative of HIV patients in general because haemophiliacs are given regular injections of Factor VIII which may be a source of bacteraemia. They are also prone to bleeding around the joint which may predispose them to infection.
We do not yet know whether HIV may influence the incidence of aseptic loosening by altering the inflammatory response to wear debris.

Although the rate of dislocation in the hips in our series was only $2.7 \%$ ( 2 of 73 ), we are concerned as to the possibility of dislocation in view of the use of squat toilets, cultural kneeling for women and subsistence farming which involves manual hoeing. Most of the current patients are middle to upper class with facilities for flush toilets and are not subsistence farmers. On discharge from hospital a toilet 'raise' modification to sit on is made for those who have squat toilets.

The aims of an African Hip Registry are different from those of a western Registry since comparison between implants and techniques are not the main issue. Consideration of the different health, social and economic settings, the impact of HIV disease and high activity are of particular interest. We have encouraged other countries in our region to establish Joint Registries. By pooling data, conclusions regarding outcome may be arrived at earlier. Comparative registries will also highlight weaknesses and help to focus training or the adjustment of clinical practice and thus optimise good clinical governance for joint replacement in the region.

We thank The British Orthopaedic Association for funding this project.

No benefits in any form have been received or will be received from a commercial party related directly or indirectly to the subject of this article.

\section{References}

1. Jellis JE. Orthopaedic surgery and HIV disease in Africa. Int Orthop 1996;20:253-6.

2. Harrison WJ, Lewis CP, Lavy CB. Wound healing after implant surgery in HIV positive patients. J Bone Joint Surg [Br] 2002;84-B:802-6.

3. Hicks JL, Ribbans WJ, Buzzard B, et al. Infected joint replacements in HIV-positive patients with haemophilia. J Bone Joint Surg [Br] 2001;83-B:1050-4.

4. Harris WH. Traumatic arthritis of the hip after dislocation and acetabular fractures: treatment by mold arthroplasty: an end-result study using a new method of result evaluation. J Bone Joint Surg [Am] 1969;5l-A:737-55.

5. Malchau H, Herberts P. Prognosis of total hip replacement: surgical and cementing technique in THR: a revision risk study at 134,056 primary operations. Scientific exhibition, 63rd AAOS Meeting. The national hip arthroplasty register.

6. Harrison WJ. HIV/AIDS in trauma and orthopaedic surgery. J Bone Joint Surg [Br] 2005;87-B:1178-81. 